COMMENT. Marijuana use may cause systemic hypotension and vasospasm, leading to CNS ischemia and infarction. The cerebellum is considered more susceptible because it lacks collateral circulation. Early diagnosis of marijuana-induced stroke is necessary to permit prompt neurosurgical relief of cerebellar edema and brainstem compression.

\title{
ENVIRONMENTAL CAUSES OF CNS MALDEVELOPMENT
}

Developmental processes and the effects of toxic agents in the environment that alter CNS growth and maturation are reviewed by a researcher in the Department of OB/GYN, University of Rochester Medical Center, Rochester, NY. Processes discussed include: establishment of neuron numbers, migration of neurons, connections, neurotransmitter activity, receptor numbers, myelin deposition, and postnatal neurogenesis. The neuroteratological effects of toxic chemicals (eg lead, methylmercury, alcohol) and new drugs (antiepileptic drugs) have been studied in relation to prenatal and early postnatal life, but little attention is given to toxins in later childhood and adolescence. Valproic acid (VPA) exposure is linked to neural tube defects, developmental delays, and possibly autism. VPA inhibits histone deacetylase, causing a change in chromatin structure and gene transcription. Ectopias can result from exposure to radiation, methylmercury, toluene, and ethanol between the $5^{\text {th }}$ and $6^{\text {th }}$ week postconception (when the cortical plate is formed) or the $5^{\text {th }}$ month postconception (when all cortical neurons have reached final migration). Genetic factors control migration: eg Reelin is critical for normal migration. Lack of normal Reelin protein underlies lissencephaly and cerebellar hypoplasia, and increased Reelin expression is reported in polymicrogyria. Reelin expression is thyroid hormone dependent. Any exposure that changes hormone or transmitter levels (eg psychoactive drugs, pesticides, cocaine, nicotine) can be teratogenic. Myelin deposition is impaired by malnutrition. Pruning, the trimming back of connections, occurs after age 2 years when the number of synapses in the brain reaches a peak. Normally, synapses decrease by $40 \%$ to the adult number during adolescence. Little is known about environmental factors and alteration in pruning. Autism, which is associated with above-average head size, may be an example of a deficit in pruning. (Rodier PM. Environmental causes of central nervous system maldevelopment. Pediatrics April 2004;113:1076-1083). (Reprints: Patricia M Rodier PhD, Department of OB/GYN, University of Rochester Medical Center, Rochester, NY 14642).

COMMENT. A new classification of CNS malformations based on patterns of genetic expression integrated with descriptive morphogenesis is proposed by Sarnat HB and Flores-Sarnat L (Eur J Paediatr Neurol 2001;5:57-64; see Ped Neur Briefs 2001;15:5758). In addition to neurotoxins, infarcts acquired in fetal life or infections may disrupt development and result in heterotopias. In an overview of recent advances, Sarnat HB cites the discovery that mutations in the organizer gene Sonic Hedgehog may be involved in malformations of the embryonic neural tube (In Progress in Pediatric Neurology III, PNB Publishers, 1997;365-369). In the past 5 years several publications have described the teratogenic effects of lead, methylmercury, alcohol, nicotine, thyroid dysfunction and other environmental factors during pregnancy. Antiepileptic drugs (AEDs) pose a continuing threat to the developing fetus. Teratogenic toxicity of traditional AEDs has been investigated, but the increasing use of newer AEDs requires caution and careful evaluation in younger female patients with epilepsy. 